CALT 68-2873

\title{
Search for Low-Mass Dark Matter at BABAR
}

\author{
Bertrand Echenard \\ Department of Physics, California Institute of Technology, MC 356-48 \\ Pasadena, California 91125, USA \\ echenard@caltech.edu \\ Submitted to Mod. Phys. Lett. A. \\ May 17, 2012
}

\begin{abstract}
This review briefly describes light dark matter searches performed by the BABAR experiment. Although dark matter candidates have traditionally been associated with heavy particles appearing in extensions of the Standard Model, a lighter component remains a well motivated alternative, and many scenarios of light dark matter have been recently proposed. Thanks to their large luminosities, $B$ factories offer an ideal environment to probe these possibilities, complementing searches from direct detection and satellite experiments.
\end{abstract}

Keywords: low-mass dark matter; Upsilon decays, hidden sector; B-factories.

PACS Nos.: 12.60-i ,13.25.Gw,14.80.Ec,14.80.Da

\section{Introduction}

The evidence for dark matter is now overwhelming, and the determination of its properties is one of the central tasks in current theoretical and experimental investigations. Of the vast array of dark matter candidates proposed during the last decades, the class known as Weakly Interacting Massive Particles (WIMPs) has many attractive features. Although WIMPs are traditionally associated with heavy particles appearing in models of New Physics, a lighter component remains a theoretically well-motivated alternative.

Many models have been recently proposed to accommodate light dark matter, ranging from minimal setups including a single dark matter particle to hidden sectors with arbitrary structures. Thanks to their large luminosities and lowbackground environments, $B$ factories offer an ideal environment to probe for $\mathrm{MeV}$ $\mathrm{GeV}$ dark matter, complementing searches from the LHC, direct detection and satellite experiments.

During the last decade, the BABAR experiment ${ }^{1}$ has collected more than $500 \mathrm{fb}^{-1}$ of data at the $\Upsilon(4 S), \Upsilon(3 S), \Upsilon(2 S)$ resonances and just below the $\Upsilon(4 S)$ resonance. This large dataset has been exploited to shed some light on many aspects of precision 
physics, from $C P$ violation to spectroscopy, and searches for light New Physics. In the following, we present a review of searches for low-mass dark matter at BABAR.

\section{Search for light dark matter in invisible $\Upsilon(1 S)$ decays.}

In a minimal scenario, a single dark matter field $(\chi)$ is added to the Standard Model (SM), together with a new boson that mediates SM-dark matter interactions 21344. This boson could be produced in $b \bar{b}$ annihilation, and subsequently decay to a pair of dark matter particles, contributing to the invisible width of the $\Upsilon$ mesons. In the SM, invisible $\Upsilon(1 S)$ decays proceed through the $\nu \bar{\nu}$ final state with a branching

fraction $B(\Upsilon(1 S) \rightarrow \nu \bar{\nu}) \sim\left(1 \times 10^{-5}\right)$ [5, well below the current experimental sensitivity. Assuming no flavor-changing currents, calculations based on the thermal dark matter relic density predict a rate $\Upsilon(1 S) \rightarrow \chi \bar{\chi}$ larger by one or two orders of magnitude than that of $\Upsilon(1 S) \rightarrow \nu \bar{\nu}$.

A search for dark matter in invisible $\Upsilon(1 S)$ decays has been performed at BABAR using a sample of $122 \times 10^{6} \Upsilon(3 S)$ mesons 7 . A clean sample of $\Upsilon(1 S)$ mesons is obtained by reconstructing $\Upsilon(3 S) \rightarrow \pi^{+} \pi^{-} \Upsilon(1 S)$ transitions. The signal topology consists of exactly two oppositely-charged tracks originating from the interaction point with no additional activity in the detector. The events are selected using a multivariate classifier based on variables describing the pions, the neutral energy deposited in the calorimeters and the multiplicity of $K_{L}^{0}$ candidates.

The distribution of the resulting dipion recoil mass, shown in Fig. 1, exhibits a clear peak corresponding to $\Upsilon(1 S)$ mesons, on top of a non-resonant component. In addition to signal events, a peaking background arising mainly from $\Upsilon(1 S)$ twobody decays, where the decay products escape undetected, is also present. This component, which is kinematically indistinguishable from signal events, is estimated from Monte Carlo simulations. After subtraction of the peaking background, the signal yield is found to be $-118 \pm 105 \pm 124$, where the first uncertainty is statistical and the second systematic.

Correcting for efficiency, a branching fraction $B(\Upsilon(1 S) \rightarrow$ invisible $)=(-1.6 \pm$ $1.4 \pm 1.6) \times 10^{-4}$ is obtained. Lacking evidence for such decays, a $90 \%$ confidence level Bayesian upper limit on its branching fraction is set at $3.0 \times 10^{-4}$ using priors flat in branching fraction. This result improves the best previous measurement ${ }^{8}$ by almost an order of magnitude, and sets stringent constraints on minimal light dark matter models.

\section{Search for light dark matter in radiative $\Upsilon(1 S)$ decays.}

In addition to purely invisible decays, light dark matter can be probed in radiative $\Upsilon \rightarrow \gamma \chi \bar{\chi}$ decays. The corresponding rate is suppressed by a factor $\mathcal{O}(\alpha)$ compared to that of the pure invisible decay, and a branching fraction in the range $10^{-5}-10^{-4}$ is expected 3 .

This final state is also sensitive to dark matter candidates predicted by several supersymmetric extensions of the Standard Model. Although the Minimal Super- 


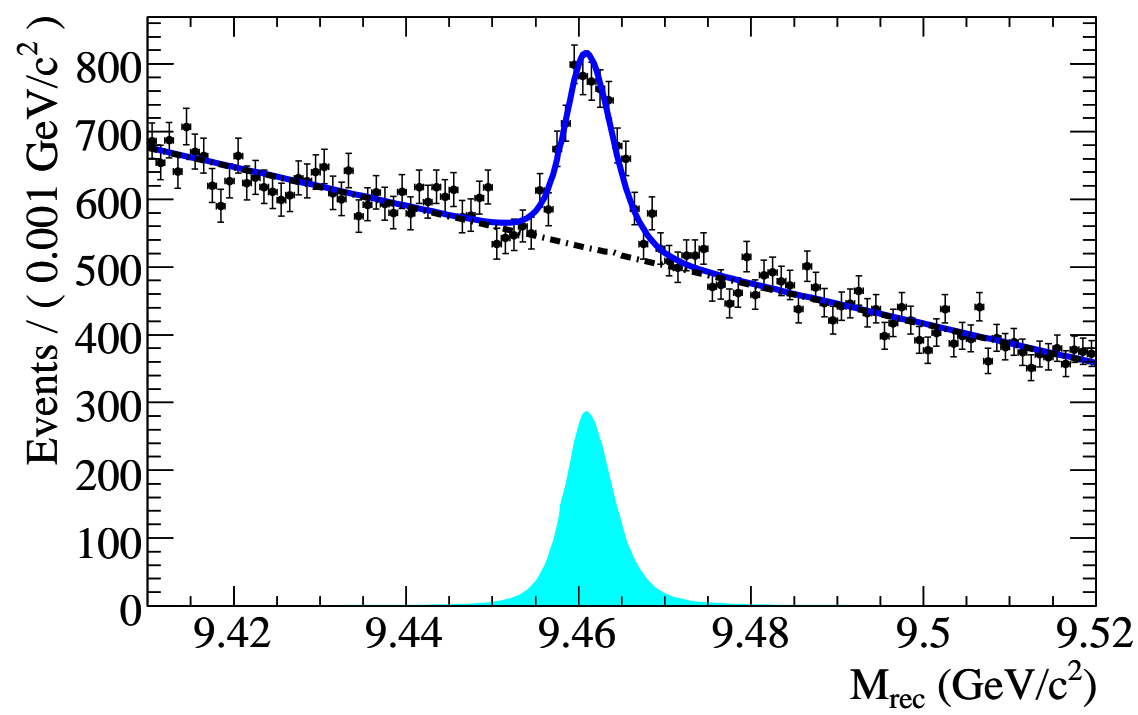

Fig. 1. The distribution of the dipion recoil mass $\left(M_{r e c}\right)$ together with the result of the maximum likelihood fit (full line). The non-resonant background (dashed line) and the sum of the signal and the peaking background (solid filled) are also shown.

symmetric Standard Model (MSSM) cannot support light dark matter, because it would require another charged or colored particle to be light as well, trivial extensions of the MSSM can easily evade these constraints. For example, the Next-toMinimal Supersymmetric Standard Model includes the possibility of a neutralino $\left(\chi_{0}\right)$ and a $C P$-odd Higgs boson $\left(A^{0}\right)$ at the $\mathrm{GeV}$ scale 9 . If kinematically allowed, the decay $\Upsilon \rightarrow \gamma+$ invisible could proceed through Wilczek production of a light scalar 10 , followed by the decay into a pair of neutralinos: $\Upsilon \rightarrow \gamma A^{0}, A^{0} \rightarrow \chi_{0} \overline{\chi_{0}}$. The branching fraction $\Upsilon \rightarrow \gamma A^{0}, A^{0} \rightarrow \chi_{0} \overline{\chi_{0}}$ is predicted to be as large as $10^{-4}-10^{-3}[9$, making it accessible to $B$ factories.

A search for this process in radiative $\Upsilon(1 S)$ decays has been performed at BABAR based on a sample of $99 \times 10^{6} \Upsilon(2 S)$ mesons 11 . The $\Upsilon(1 S)$ mesons are tagged using the dipion transition $\Upsilon(2 S) \rightarrow \pi^{+} \pi^{-} \Upsilon(1 S)$. The signal signature is a photon with two oppositely charged tracks identified as pions. The signal is extracted by a series of bidimensional unbinned likelihood fits to the dipion recoil mass and the missing mass squared. Values of $m_{\chi_{0}}$ are probed in steps of $0.1-0.5 \mathrm{GeV}$ over $0 \leq m_{\chi_{0}} \leq 4.5 \mathrm{GeV}$.

No significant signal is observed, and $90 \%$ confidence level limits on the branching fraction $B\left(\Upsilon(1 S) \rightarrow \gamma \chi_{0} \overline{\chi_{0}}\right)$ are set in the range $(0.5-24) \times 10^{-5}$ (Fig. 2), assuming a phase-space distribution for the photon energy. This result improves the best previous measurement 13 by almost two orders of magnitude, and con- 
strains on light Higgs boson and dark matter models.

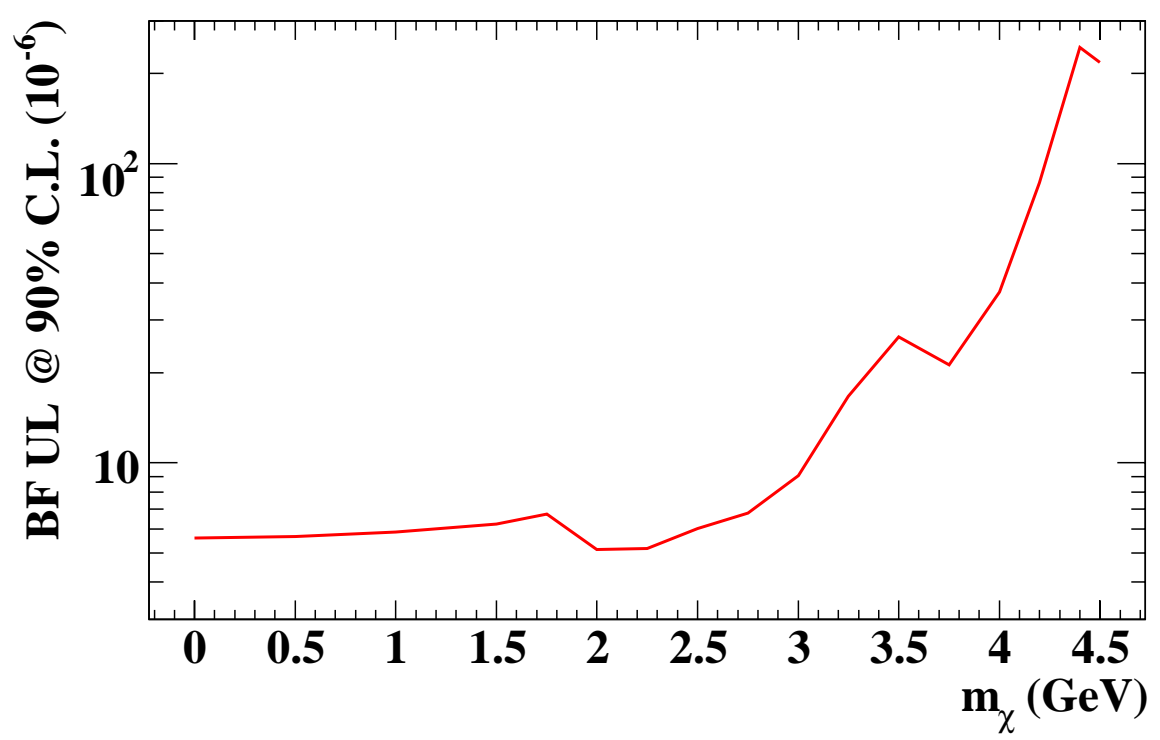

Fig. 2. $90 \%$ confidence level upper limit on the branching fraction $B\left(\Upsilon(1 S) \rightarrow \gamma \chi_{0} \overline{\chi_{0}}\right)$ as a function of $m_{\chi_{0}}$.

\section{Search for dark matter and hidden sectors}

A new class of dark matter model has recently been proposed, motivated by results from terrestrial and satellite experiments. These theories introduce a new hidden sector with WIMP-like fermionic dark matter particles charged under a new Abelian gauge group 14|15|16. The corresponding gauge boson, dubbed a hidden photon $\left(A^{\prime}\right)$, couples to SM fermions through its kinetic mixing with the SM photon 17 . The hidden photon mass is constrained to be at most a few $\mathrm{GeV}$ to be compatible with the electron/positron excess observed by PAMELA 18 and FERMI 19]20, without a comparable anti-proton signal.

The hidden boson masses are usually generated via the Higgs mechanism, adding hidden Higgs bosons $\left(h^{\prime}\right)$ to the theory. A minimal hidden sector model includes a single hidden photon and a Higgs boson 21. Additional Higgs and gauge bosons appear in more complex setups 22. The coupling strength between the SM and the hidden sector, $\epsilon$, can naturally be as large as $10^{-4}-10^{-2} 21$, opening the possibility of probing a light hidden sector at low-energy $e^{+} e^{-}$colliders. 


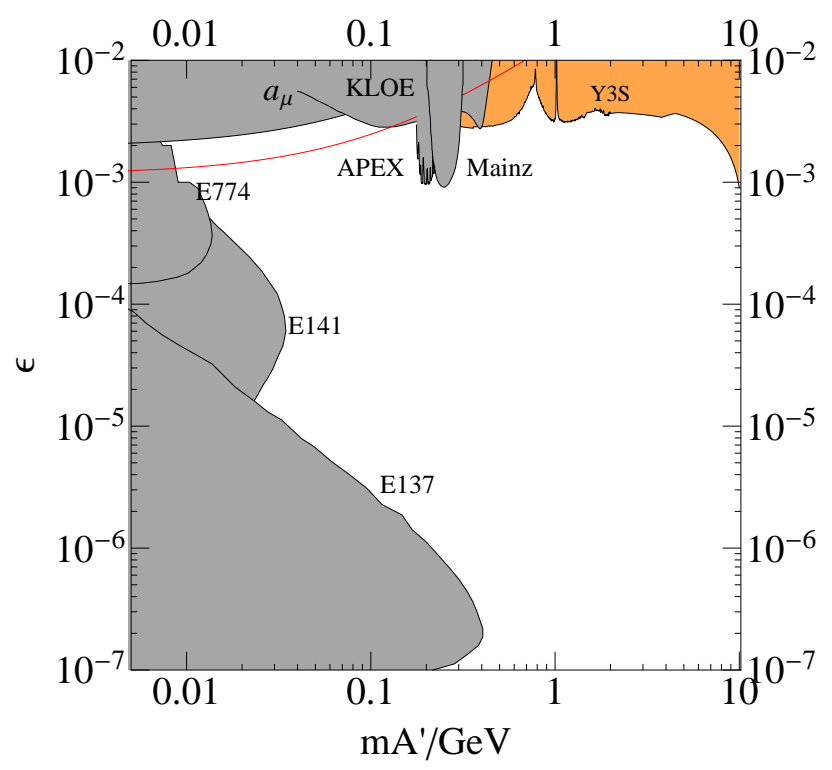

Fig. 3. Constraints on the mixing parameters, $\epsilon$, as a function of the hidden photon mass derived from searches in $\Upsilon(2 S, 3 S)$ decays at BABAR (orange shading) and from other experiments $24|25| 26$ (gray shading). The red line shows the value of the coupling required to explain the discrepancy between the calculated and measured anomalous magnetic moment of the muon 30 .

\subsubsection{Search for a hidden photon}

Hidden photons can be readily produced in $e^{+} e^{-} \rightarrow \gamma A^{\prime}, A^{\prime} \rightarrow l^{+} l^{-}(l=e, \mu)$ interactions, and be detected as resonances in the $l^{+} l^{-}$spectrum. This signature is similar to that of light $C P$-odd Higgs production in $e^{+} e^{-} \rightarrow \Upsilon(2 S, 3 S) \rightarrow \gamma \mu^{+} \mu^{-23}$, and searches for this final state have been reinterpreted as searches for hidden photon production 24 . These limits are shown in Fig. 3, together with the constraints derived from the measurement of the muon anomalous magnetic moment, and searches at low-energy $e^{+} e^{-}$colliders and fixed-target experiments 24|25|26. Values of the mixing strength down to $10^{-3}-10^{-2}$ are excluded for $0.212<m_{A^{\prime}}<9.3 \mathrm{GeV}$. Future analyses of the $e^{+} e^{-} \rightarrow \gamma l^{+} l^{-}(l=e, \mu)$ final states are expected to probe values of the mixing strength down to $10^{-3}$ in the range $0.01<m_{A^{\prime}}<10 \mathrm{GeV}$, significantly improving existing bounds.

Other measurements may be reinterpreted as constraints on hadronic hidden photon decays. These include searches for peaks in inclusive $e^{+} e^{-} \rightarrow \gamma$ hadrons production 27, as well as exclusive hadronic final states, such as $e^{+} e^{-} \rightarrow \gamma \pi^{+} \pi^{-} 28$. Invisible decays, which occur if all hidden sector states decay to long-lived particles, could also be detected as a mono-energetic photon signature in $e^{+} e^{-} \rightarrow \gamma$ invisible events 29 . 


\subsubsection{Search for hidden gauge bosons}

Extensions of hidden sectors to a non-Abelian groups introduce additional hidden gauge bosons to the theory, generically denoted $W^{\prime}, W^{\prime \prime}, \ldots$ The detailed phenomenology depends on the structure of the model, but heavy hidden bosons decay to lighter hidden states if kinematically accessible, while the lightest bosons are metastable and decay to SM fermions via their mixing with the hidden photon 22 .

A pair of light hidden bosons can be produced via an off-shell $A^{\prime}$ in $e^{+} e^{-} \rightarrow$ $A^{\prime *} \rightarrow W^{\prime} W^{\prime \prime}$ collisions. BABAR performed a search for di-boson production using $513 \mathrm{fb}^{-1}$ of data, collected mostly at the $\Upsilon(4 S)$ resonance $\underline{31}$. The bosons are reconstructed via their decay into muons or electrons. It is furthermore assumed that their mass is nearly degeneratea, and their width is negligible compared to the detector resolution.

The signature consists of four leptons with zero total charge carrying the full beam energy, forming two narrow dileptonic resonances with similar masses and width consistent with the experimental resolution. This topology is quite unique; the only backgrounds arise from QED processes.

The signal is extracted as a function of the average dileptonic mass in the range $0.24-5.3 \mathrm{GeV}$ in $10 \mathrm{MeV}$ steps. No significant signal is observed, and limits on the $e^{+} e^{-} \rightarrow A^{\prime *} \rightarrow W^{\prime} W^{\prime \prime}$ cross-section are set, assuming the two bosons have nearly degenerate masses and equal branching fractions into electrons and muons. The results are translated into limits on the product $\alpha_{D} \epsilon^{2}$, where $\alpha_{D}=g_{D}^{2} / 4 \pi$ and $g_{D}$ is the hidden sector gauge coupling constant. Values down to $10^{-10}$ can be probed. Assuming $\alpha_{D}=\alpha \simeq 1 / 137$, limits on the mixing strength at the level of $10^{-4}-10^{-2}$ are set for this process.

\subsubsection{Search for hidden Higgs bosons}

The Higgsstrahlung process, $e^{+} e^{-} \rightarrow A^{\prime} h^{\prime}, h^{\prime} \rightarrow A^{\prime} A^{\prime}$, offers another promising gateway to hidden sectors, since it is one of the few processes suppressed by a single power of the mixing strength, and the background is expected to be small. The event topology depends on the boson mass: Higgs bosons heavier than two hidden photons decay promptly, while their lifetime becomes large enough to escape undetected in the regime $m_{h^{\prime}}<m_{A^{\prime}}$.

$B A B A R$ performed a search for a hidden Higgs boson in Higgsstrahlung production in the prompt decay regime based on $521 \mathrm{fb}^{-1}$ of data 32 . The measurement is performed in the range $0.8<m_{h^{\prime}}<10.0 \mathrm{GeV}$ and $0.25<m_{A^{\prime}}<3.0 \mathrm{GeV}$, with the constraint $m_{h^{\prime}}>2 m_{A^{\prime}}$. The signal is either fully reconstructed into lepton or pion pairs (exclusive mode), or partially reconstructed (inclusive mode). Only two of the three hidden photons are reconstructed in the latter case, and the four-momentum of the remaining hidden photon is identified to that of the recoiling system. The topology of the exclusive modes consists of six tracks having an invariant mass close

a The production of identical bosons is forbidden by the Landau-Yang theorem 
to the center-of-mass energy, forming three hidden photon candidates with equal masses. Inclusive modes are first identified by selecting two dileptonic resonances with similar mass, and requiring the mass of the recoiling system to be compatible with the Higgsstrahlung hypothesis.

No significant signal is observed. Using uniform priors in the cross-section, upper limits on the $e^{+} e^{-} \rightarrow A^{\prime} h^{\prime}, h^{\prime} \rightarrow A^{\prime} A^{\prime}$ cross section are derived as a function of the hidden Higgs and hidden photon masses, including systematic uncertainties.

These results are translated into limits on the product $\alpha_{D} \epsilon^{2}$, and displayed in Fig. 4 as a function of the hidden photon mass for selected values of the hidden Higgs boson mass. Values down to $10^{-10}-10^{-8}$ are excluded for a large range of hidden photon and hidden Higgs masses, assuming prompt decays. Assuming $\alpha_{D}=\alpha \simeq 1 / 137$, limits on the mixing strength in the range $10^{-4}-10^{-3}$ are derived, an order of magnitude smaller than the current experimental bounds extracted from direct photon production in this mass range.

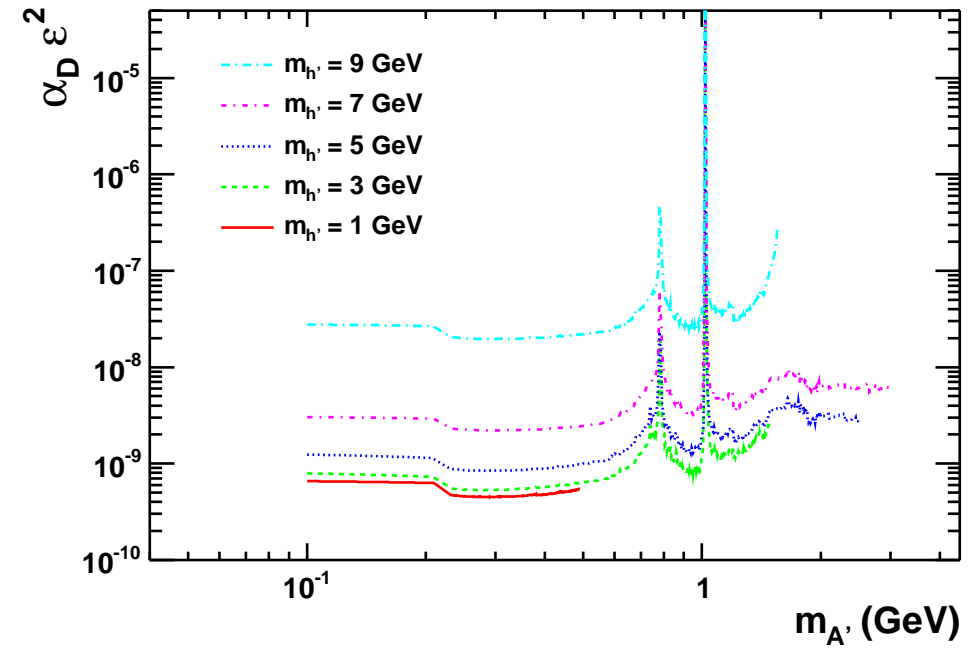

Fig. 4. The $90 \%$ confidence level upper limit on the product $\alpha_{D} \epsilon^{2}$ as a function of the hidden photon mass $\left(m_{A^{\prime}}\right)$ for selected values of hidden Higgs boson masses $\left(m_{h^{\prime}}\right)$.

\subsection{Conclusion}

Recent evidence has suggested that dark matter might contain a $\mathrm{MeV}-\mathrm{GeV}$ scale component. Thanks to their large luminosities, $B$ factories provide an ideal environment to probe for such a possibility, complementing direct detection and satellite experiments. No sign of light dark matter has been observed so far, and constraints on theoretical models have been set, significantly improving bounds from previous experiments. Super flavor factories are expected to improve the sensitivity of these 
searches by a factor $10-100$, further constraining the parameter space of these theories. Many more results are to come in the near future, which will hopefully shed some light on the nature of dark matter.

\section{Acknowledgments}

I would like to thank David Hitlin and Yury Kolomensky for their comments on this manuscript, and Rouven Essig for useful theoretical discussions. I am also grateful to Matthew Graham for discussing the constraints on dark photon production and providing the corresponding figure. BE is supported by the Department of Energy, under grant DE-FG02-92ER40701.

\section{References}

1. B. Aubert et al. [BABAR Collaboration], Nucl. Instrum. Meth. A 479, 1 (2002).

2. B. McElrath, Phys. Rev. D 72, 103508 (2005).

3. G. K. Yeghiyan, Phys. Rev. D 80, 115019 (2009).

4. P. Fayet, Phys. Rev. D 81, 054025 (2010).

5. L. N. Chang, O. Lebedev and J. N. Ng, Phys. Lett. B 441, 419 (1998).

6. B. McElrath, eConf C 070805, 19 (2007).

7. B. Aubert et al. [BABAR Collaboration], Phys. Rev. Lett. 103, 251801 (2009).

8. O. Tajima et al. [Belle Collaboration], Phys. Rev. Lett. 98, 132001 (2007).

9. J. F. Gunion, D. Hooper and B. McElrath, Phys. Rev. D 73, 015011 (2006).

10. F. Wilczek, Phys. Rev. Lett. 39, 1304 (1977).

11. P. del Amo Sanchez et al. [BABAR Collaboration], Phys. Rev. Lett. 107 (2011) 021804.

12. S. B. Athar et al. [CLEO Collaboration], Phys. Rev. D 73, 032001 (2006).

13. R. Balest et al. [CLEO Collaboration], Phys. Rev. D 51, 2053 (1995).

14. P. Fayet et. al., Phys. Rev. D 75 (2007) 115017, and references therein;

15. M. Pospelov, A. Ritz and M. B. Voloshin, Phys. Lett. B 662, 53 (2008).

16. N. Arkani-Hamed, D. P. Finkbeiner, T. R. Slatyer and N. Weiner, Phys. Rev. D 79, 015014 (2009).

17. B. Holdom, Phys. Lett. B 166, 196 (1985).

18. O. Adriani et al. [PAMELA Collaboration], Nature 458, 607 (2009).

19. A. A. Abdo et al. [Fermi LAT Collaboration], Phys. Rev. Lett. 102, 181101 (2009).

20. M. Ackermann et al. [Fermi LAT Collaboration], Phys. Rev. D 82, 092004 (2010).

21. B. Batell, M. Pospelov and A. Ritz, Phys. Rev. D 79, 115008 (2009).

22. R. Essig, P. Schuster and N. Toro, Phys. Rev. D 80, 015003 (2009).

23. B. Aubert et al. [BABAR Collaboration], Phys. Rev. Lett. 103, 081803 (2009).

24. J. D. Bjorken, R. Essig, P. Schuster and N. Toro, Phys. Rev. D 80,075018 (2009) and references therein.

25. S. Giovannella, J. Phys. Conf. Ser. 335, 012067 (2011).

26. S. Abrahamyan et al. [APEX Collaboration], Phys. Rev. Lett. 107, 191804 (2011).

27. J. P. Lees et al. [BABAR Collaboration], Phys. Rev. Lett. 107, 221803 (2011).

28. B. Aubert et al. [BABAR Collaboration], Phys. Rev. Lett. 103 (2009) 231801.

29. B. Aubert et al. [BABAR Collaboration], arXiv:0808.0017 [hep-ex].

30. M. Pospelov, Phys. Rev. D 80, 095002 (2009).

31. B. Aubert et al. [BABAR Collaboration], arXiv:0908.2821 [hep-ex].

32. J. P. Lees [BABAR Collaboration], arXiv:1202.1313 [hep-ex]. 